\title{
Bulk Assemblies of Lead Bromide Trimer Clusters with Geometry Dependent Photophysical Properties
}

Sujin Lee,${ }^{\dagger}$ Chenkun Zhou,${ }^{\ddagger}$ Jennifer Neu,,${ }^{\curvearrowleft}$ Drake Beery,,${ }^{\dagger}$ Ashley Arcidiacono, ${ }^{\dagger}$ Maya Chaaban,,${ }^{\dagger}$

Haoran Lin, ${ }^{\dagger}$ Alyssa Gaiser,${ }^{\dagger}$ Banghao Chen,${ }^{\dagger}$ Thomas E Albrecht-Schmitt, ${ }^{\dagger}$ Theo Siegrist,${ }^{\ddagger} \uparrow$ and Biwu $\mathrm{Ma}^{\dagger,+}$

${ }^{\dagger}$ Department of Chemistry and Biochemistry, Florida State University, Tallahassee, Florida 32306, United States

\$Department of Chemical and Biomedical Engineering, FAMU-FSU College of Engineering, Tallahassee, Florida 32310, United States

๑National High Magnetic Field Laboratory, Florida State University, Tallahassee, Florida 32310, United States

Analysis of octahedral distortion. The degree of octahedral distortions was determined by using the following parameters. ${ }^{1}$

$$
\text { Bond length distortion: } \Delta_{o c t}=\frac{1}{6} \sum\left(\frac{d_{i}-d_{\text {avg }}}{d_{\text {avg }}}\right)^{2} \text {, }
$$

$d_{\mathrm{avg}}$ : the mean $\mathrm{Pb}-\mathrm{X}$ bond distance, $d_{\mathrm{i}}$ : the distances of six $\mathrm{Pb}-\mathrm{X}$ bond;

$$
\text { Halide distance deviation: } D I(\mathrm{X}-\mathrm{X})=\frac{\sum\left|(X-X)_{i}-(X-X)_{\text {avg }}\right|}{12 \times(X-X)_{\text {avg }}} \text {, }
$$

$(X-X)_{\mathrm{i}}$ : octahedral edge length, $(X-X)_{\mathrm{avg}}$ : average octahedral edge length;

$$
\text { Octahedral elongation: } \lambda_{o c t}=\frac{1}{6} \sum\left(\frac{d_{i}}{d_{0}}\right)^{2} \text {, }
$$

$d_{0}$ : the center-to-vertex distance of a regular polyhedron of the same volume;

$$
\text { Octahedral angle variance: } \sigma_{o c t}^{2}=\frac{1}{11} \sum\left(\alpha_{i}-90\right)^{2} \text {, }
$$

$\alpha_{\mathrm{i}}: \mathrm{X}-\mathrm{Pb}-\mathrm{X}$ angles;

$$
\text { Volume discrepancy: } V(\%)=\left(V_{\text {avg }}-V_{o c t}\right) \times \frac{100}{V_{\text {avg }}} \text {, }
$$

$V_{\text {oct }}$ : the volume of the octahedral $\mathrm{PbBr}_{6}, V_{\text {avg }}$ : the volume of the ideal octahedral with the edge length equal to $(X-X)_{\text {avg. }}$. 


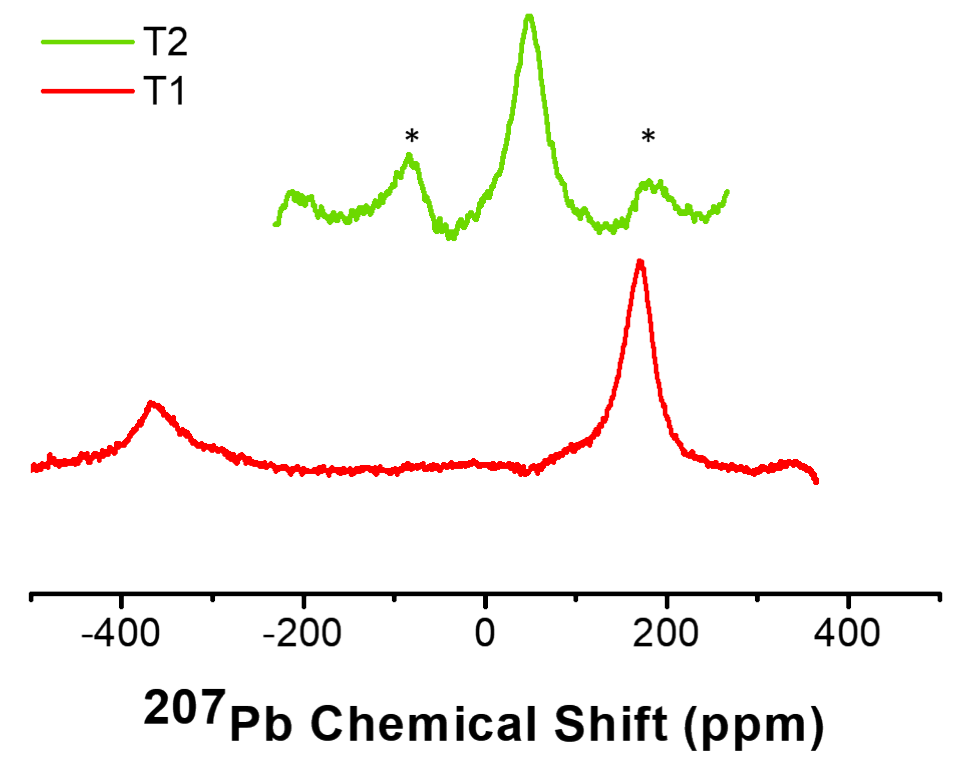

Figure S1. ${ }^{207} \mathrm{~Pb}$ NMR spectra of (bmpy) $)_{6}\left[\mathrm{~Pb}_{3} \mathrm{Br}_{12}\right](\mathbf{T 1})$ and (bmpy) ${ }_{9}\left[\mathrm{ZnBr}_{4}\right]_{2}\left[\mathrm{~Pb}_{3} \mathrm{Br}_{11}\right](\mathbf{T 2})$ (* denotes spinning side bands).

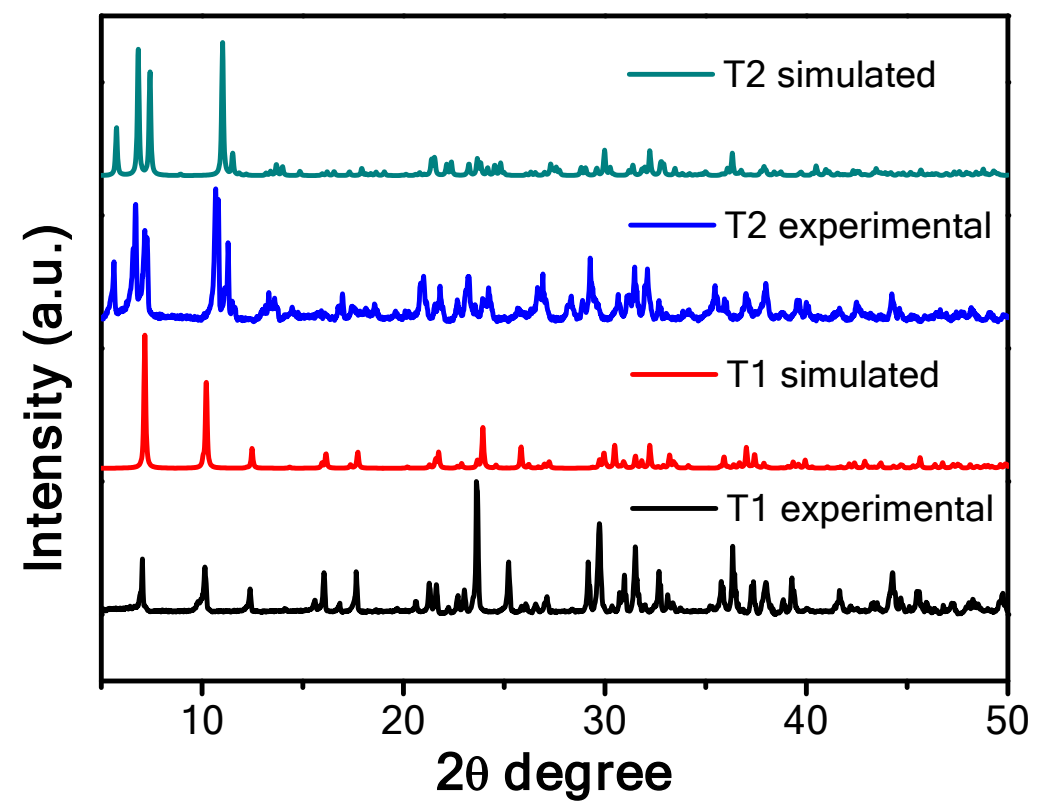

Figure S2. PXRD patterns of (bmpy) $)_{6}\left[\mathrm{~Pb}_{3} \mathrm{Br}_{12}\right](\mathbf{T 1})$ and (bmpy) $)_{9}\left[\mathrm{ZnBr}_{4}\right]_{2}\left[\mathrm{~Pb}_{3} \mathrm{Br}_{11}\right](\mathbf{T} 2)$ and its $\mathrm{SCXRD}$ simulate result. 


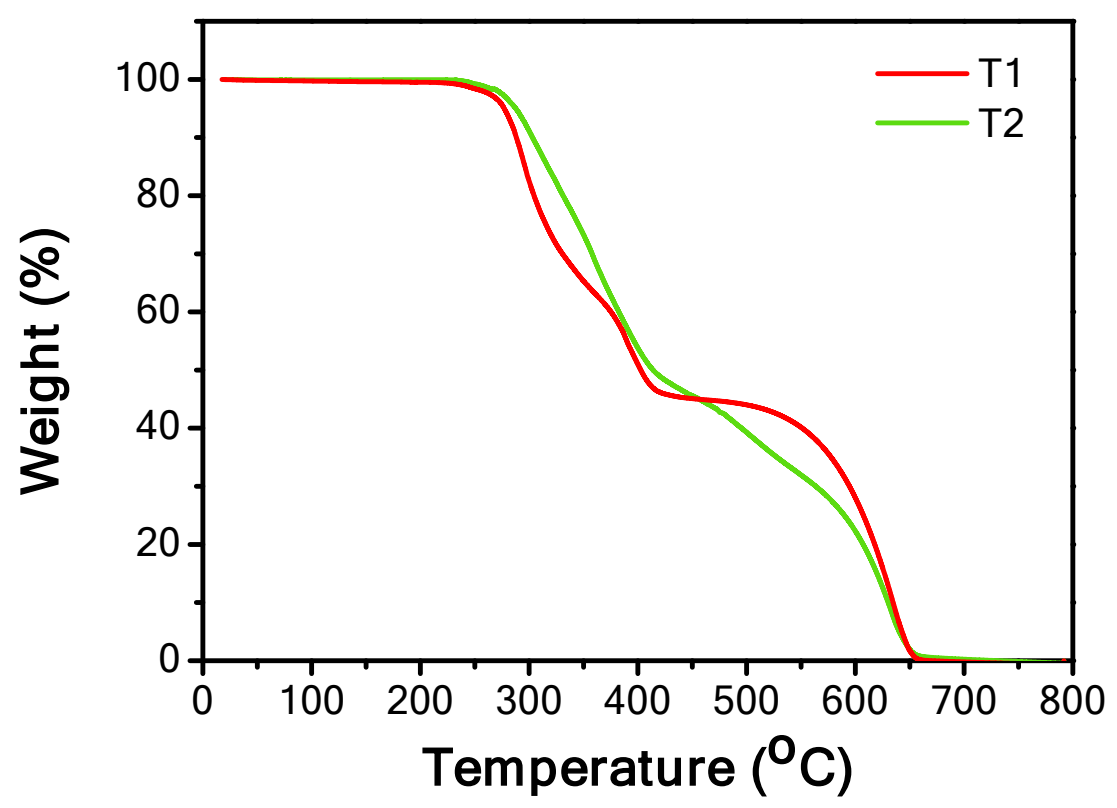

Figure S3. TGA analysis of (bmpy) $)_{6}\left[\mathrm{~Pb}_{3} \mathrm{Br}_{12}\right](\mathbf{T 1})$ and (bmpy) $)_{9}\left[\mathrm{ZnBr}_{4}\right]_{2}\left[\mathrm{~Pb}_{3} \mathrm{Br}_{11}\right](\mathbf{T} \mathbf{2})$.

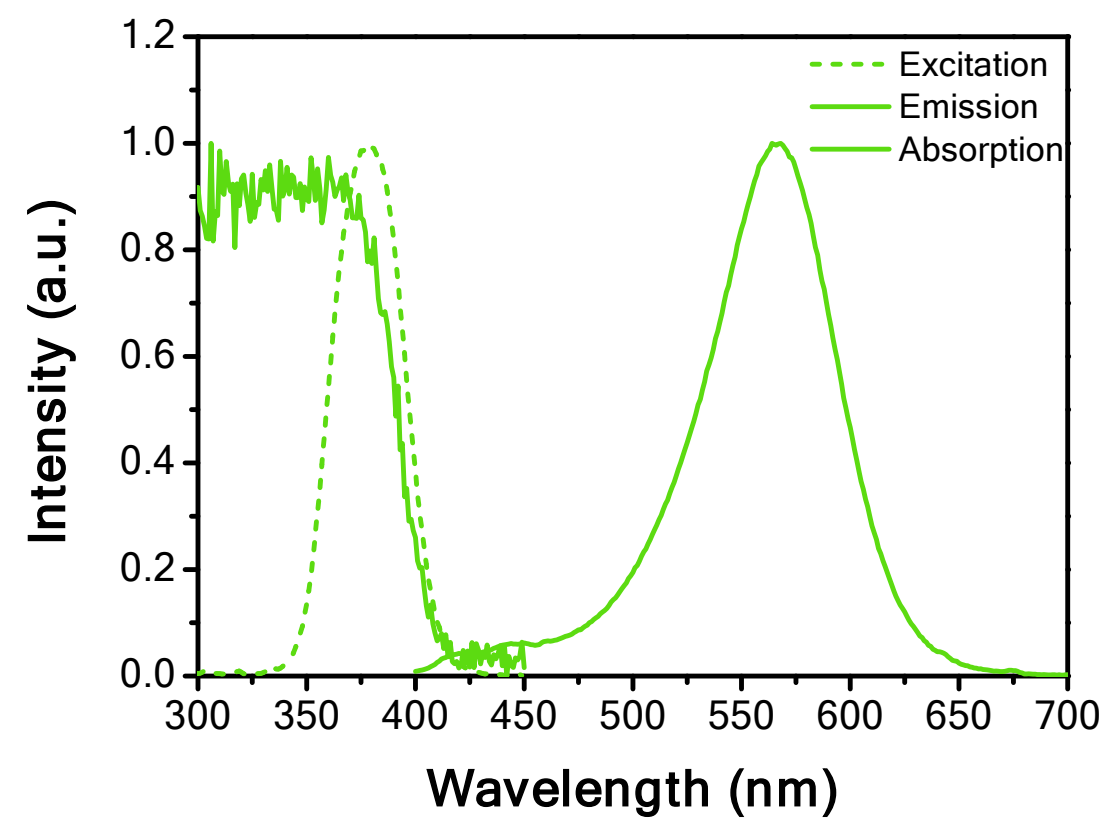

Figure S4. Photophysical properties of (bmpy) $)_{9}\left[\mathrm{ZnBr}_{4}\right]_{2}\left[\mathrm{~Pb}_{3} \mathrm{Br}_{11}\right](\mathbf{T} 2)$ at room temperature. 


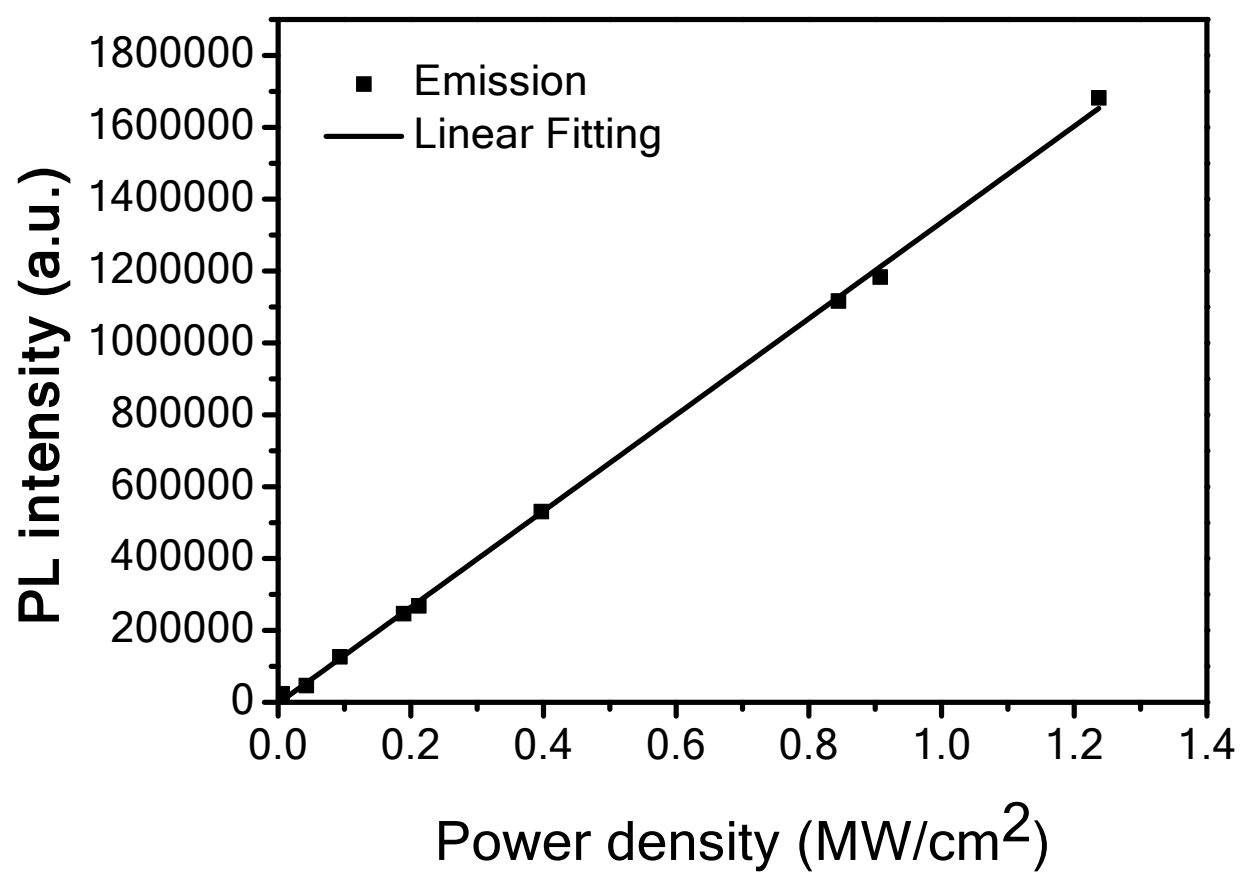

Figure S5. The emission intensity of photoluminescence plotted as a function of excitation power density at room temperature for $\mathbf{T} 2$.

(A)

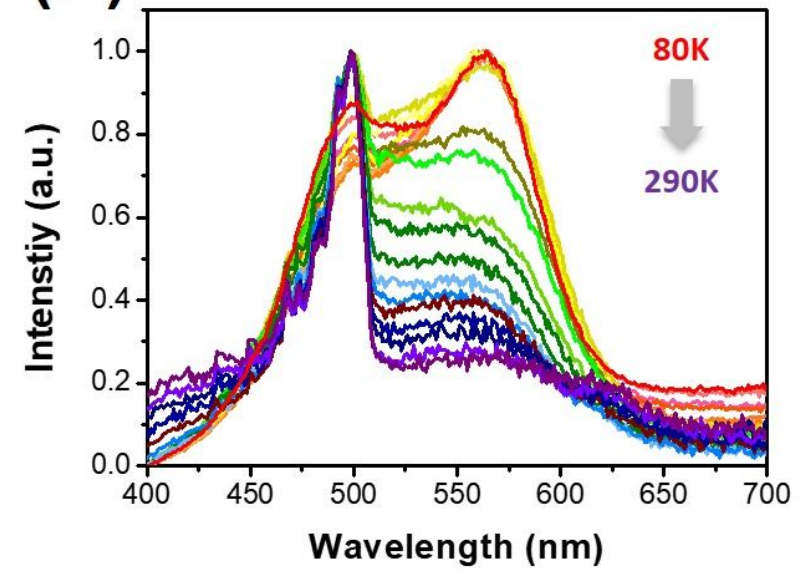

(B)

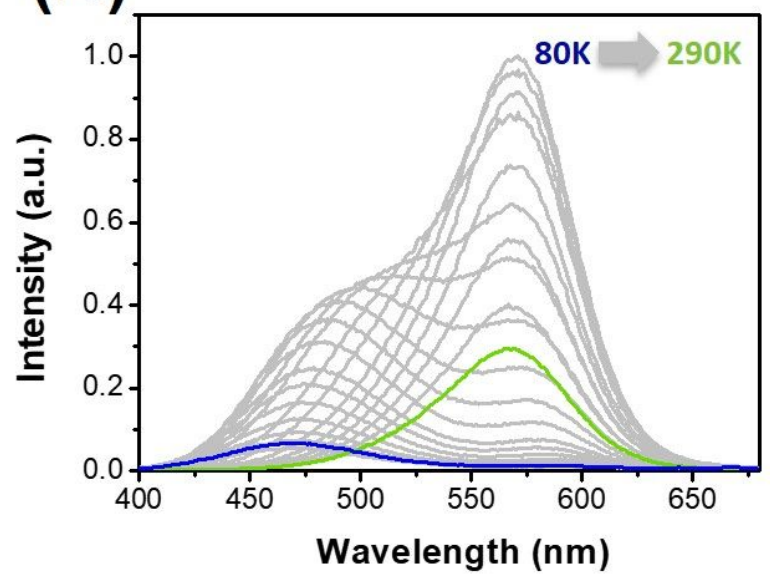

Figure S6. Temperature dependent emission spectra of T1 (A) and T2 (B) from $80 \mathrm{~K}$ to room temperature $(10 \mathrm{~K} / \mathrm{scan})$. 


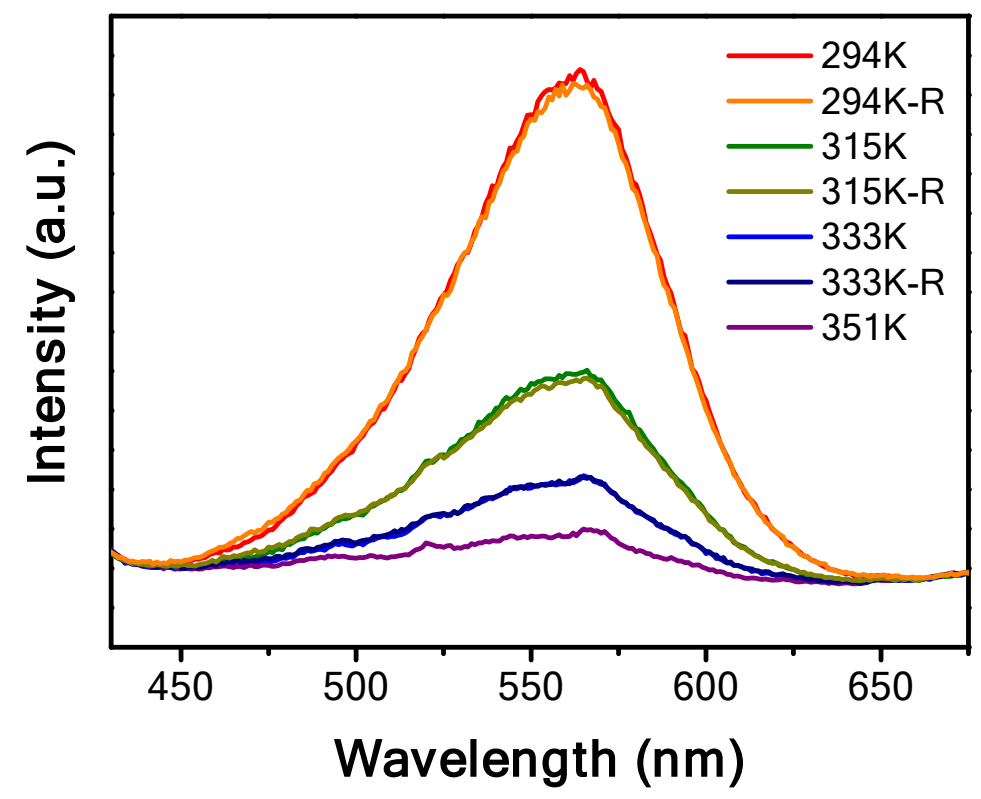

Figure S7. Temperature dependent photoluminescence from room temperature to $353 \mathrm{~K}$ for (bmpy) $)_{9}\left[\mathrm{ZnBr}_{4}\right]_{2} \mathrm{~Pb}_{3} \mathrm{Br}_{11}(\mathbf{T} 2)$.

(A)

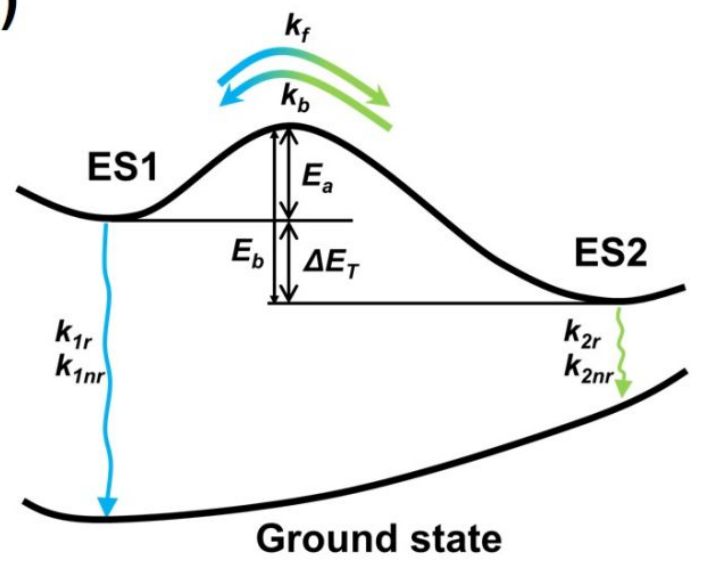

(B)

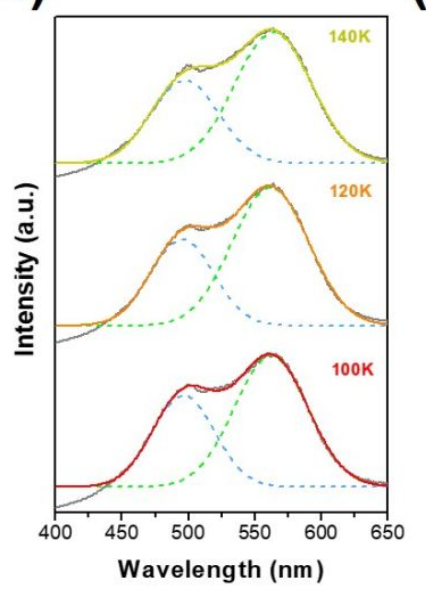

(C)

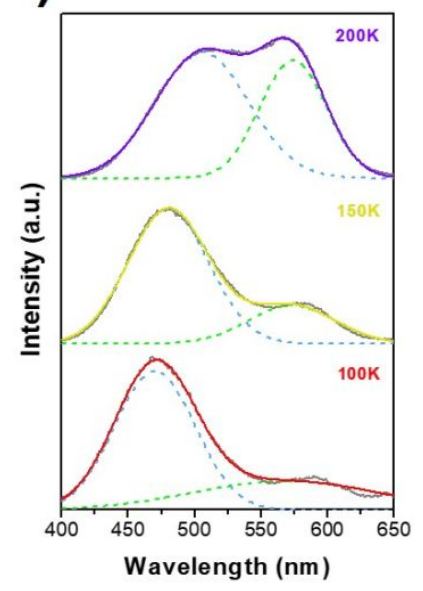

Figure S8. (A) Schematic representation of photophysical processes for T1 and T2. $E_{a}$ and $E_{b}$ are the energy barriers, $\Delta \mathrm{E}_{\mathrm{T}}$ is the energy difference between the ES1 and ES2 states, $\mathrm{k}_{\mathrm{f}}$ is the rate of excited state dynamics from ES1 to ES2, $\mathrm{k}_{\mathrm{b}}$ is the rate of reverse excited state dynamics, $\mathrm{k}_{1 \mathrm{r}}$ and $\mathrm{k}_{2 \mathrm{r}}$ are the radiative decay rates of the blue and green emissions, $\mathrm{k}_{1 \mathrm{nr}}$ (without accounting for $\mathrm{k}_{\mathrm{f}}$ ) and $\mathrm{k}_{2 \mathrm{nr}}$ (without accounting for $\mathrm{k}_{\mathrm{b}}$ ) are the non-radiative decay rates for ES1 and ES2. (B) Emission spectra of T1 fitted by the combination of Gaussian shaped blue and green emissions at different temperature. (C) Emission spectra of $\mathbf{T} 2$ fitted by the combination of Gaussian shaped blue and green emissions at different temperatures. ${ }^{2}$ 


\section{$\underline{\text { Derivation of Equations }}$}

$$
\begin{gathered}
k_{f}=A e^{\frac{-E_{a}}{R T}} \\
k_{b}=A e^{\frac{-E_{b}}{R T}} \\
\mathrm{r}=k_{f} C(E S 1)-k_{b} C(E S 2)
\end{gathered}
$$

where, $\mathrm{A}$ is the pre-exponential factor, $\mathrm{E}_{\mathrm{a}}$ is the energy barrier going from ES1 to ES2, $\mathrm{E}_{\mathrm{b}}$ is the energy barrier going from ES2 to ES1, and R is the universal gas constant.

When it reaches equilibrium, $r=0$ :

$$
\begin{gathered}
\frac{C(E S 1)}{C(E S 2)}=\frac{k_{b}}{k_{f}} \\
\eta_{1}=\frac{k_{1 r}}{k_{1 r}+k_{1 n r}} \times \frac{k_{b}}{k_{f}+k_{b}} \\
\eta_{2}=\frac{k_{2 r}}{k_{2 r}+k_{2 n r}} \times \frac{k_{f}}{k_{f}+k_{b}} \\
\frac{\eta_{2}}{\eta_{1}}=\frac{k_{f}}{k_{b}} \times \frac{k_{2 r}}{k_{1 r}} \times \frac{k_{1 r}+k_{1 n r}}{k_{2 r}+k_{2 n r}} \\
\frac{-\left(E_{a}-E_{b}\right)}{\eta_{2}} \times \frac{k_{2 r}}{k_{1 r}} \times \frac{k_{1 r}+k_{1 n r}}{k_{2 r}+k_{2 n r}} \\
\eta_{1}=A e^{\frac{-\Delta E_{T}}{R T}} \times \frac{k_{2 r}}{k_{1 r}} \times \frac{k_{1 r}+k_{1 n r}}{k_{2 r}+k_{2 n r}}
\end{gathered}
$$

where, $\frac{\eta_{2}}{\eta_{1}}$ is the green/blue emission intensity ratio.

Since $\quad k_{m r}=\frac{\eta_{m}}{t_{m}}, m=1$ or 2 if we assume $\left(\eta_{m}+\eta_{n m r}\right) \propto \eta_{m}$

The correlations between the intensity ratio and the energy difference between the ES1 and ES2 states can be described as,

$$
\frac{\eta_{2}}{\eta_{1}}=A e^{\frac{-\Delta E_{T}}{R T}}
$$

Here intensities at different temperatures were used to estimate the ratio of ES2 to ES1, and the relationship between Ratio (ES2/ES1) and 1/T were observed: 

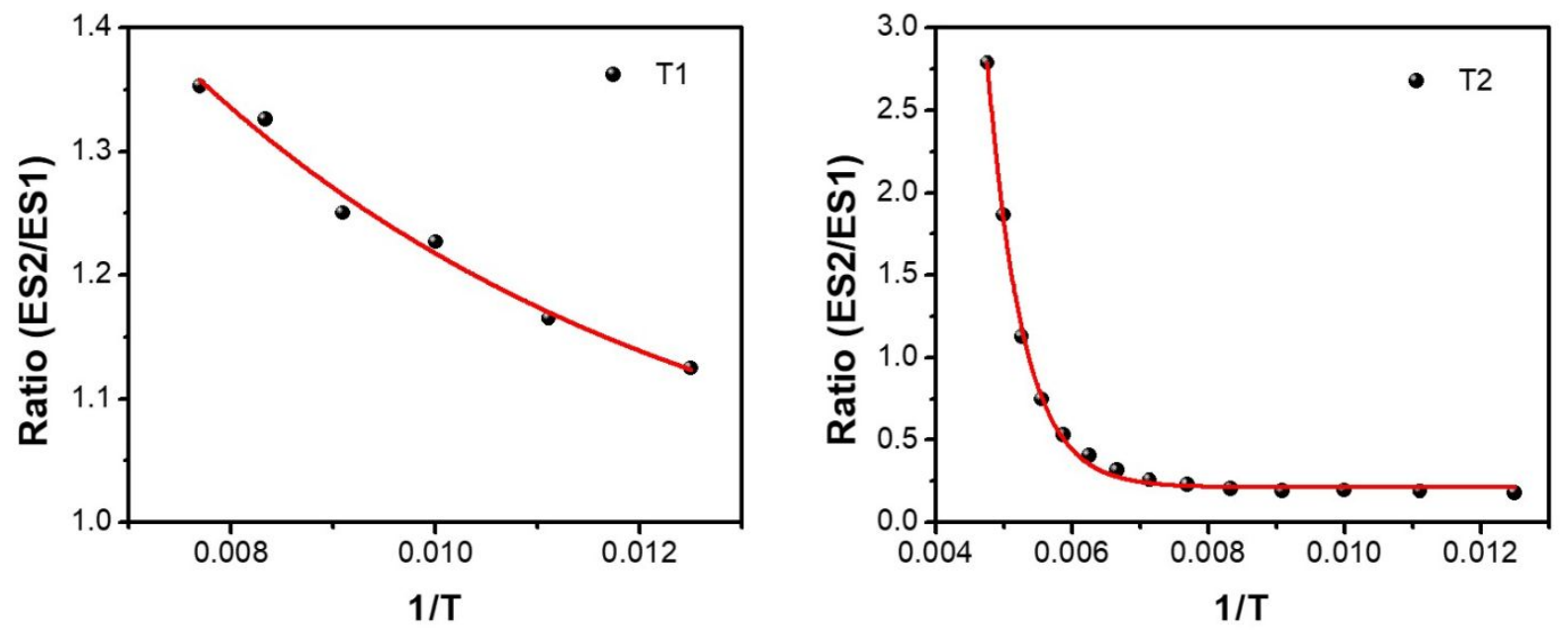

Therefore, $\Delta \mathrm{E}_{\mathrm{T}}$ were calculated to be $5 \times 10^{-8} \mathrm{eV}$ and $0.166 \mathrm{eV}$ for T1 and T2. Meanwhile, dual emission presents in $\mathbf{T} 1$ throughout the temperature window between $80 \mathrm{~K}$ and room temperature, suggesting both energy barriers of $E_{a}$ and $E_{b}$ could be overcome by thermal energy even at low temperature (80K), which would be in the order of $0.007 \mathrm{eV}$. In T2, $E_{b}$ is be much larger than $E_{a}\left(E_{b}=E_{a}+0.166 \mathrm{eV}\right)$, and $E_{a}$ has a moderate value in the order of $0.025 \mathrm{eV}$, which is hard to be overcome by thermal energy at low temperature $(80 \mathrm{~K})$, but could be overcome at room temperature $(0.025 \mathrm{eV})$.

Table S1. Single crystal x-ray diffraction data and collection parameters of (bmpy) $6\left[\mathrm{~Pb}_{3} \mathrm{Br}_{12}\right]$ and (bmpy) ${ }_{9}\left[\mathrm{ZnBr}_{4}\right]_{2}\left[\mathrm{~Pb}_{3} \mathrm{Br}_{11}\right]$; the collection was taken at a temperature of $100 \mathrm{~K}$ and $110 \mathrm{~K}$.

\begin{tabular}{lll}
\hline & $(\text { bmpy })_{6}\left[\mathrm{~Pb}_{3} \mathrm{Br}_{12}\right](\mathbf{T 1})$ & $(\text { bmpy })_{9}\left[\mathrm{ZnBr}_{4}\right]_{2}\left[\mathrm{~Pb}_{3} \mathrm{Br}_{11}\right](\mathbf{T} 2)$ \\
\hline Formula & $\left(\mathrm{C}_{9} \mathrm{NH}_{20}\right)_{6} \mathrm{~Pb}_{3} \mathrm{Br}_{12}$ & $\left(\mathrm{C}_{9} \mathrm{NH}_{20}\right)_{9}\left[\mathrm{ZnBr}_{4}\right]_{2}\left[\mathrm{~Pb}_{3} \mathrm{Br}_{11}\right]$ \\
Molecular weight & $2434.03 \mathrm{~g} / \mathrm{mol}$ & $3550.91 \mathrm{~g} / \mathrm{mol}$ \\
Space group & $\mathrm{R}-3(\# 148)$ & $\mathrm{P} 6_{3}(\# 173)$ \\
a & $17.3118(7) \AA$ & $14.9238(3) \AA$ \\
b & $17.3118(7) \AA$ & $14.9238(3) \AA$ \\
c & $21.7019(8) \AA$ & $30.6972(7) \AA$ \\
$\alpha$ & $90^{\circ}$ & $90^{\circ}$ \\
$\beta$ & $90^{\circ}$ & $90^{\circ}$ \\
$\gamma$ & $120^{\circ}$ & $120^{\circ}$ \\
$\mathrm{V}$ & $5632.7(5) \AA$ & $5920.9(2) \AA^{3}$ \\
$\mathrm{Z}$ & 3 & 2 \\
$\rho_{\text {calc. }}$ & $2.153 \mathrm{~g} / \mathrm{cm}^{3}$ & $1.992 \mathrm{~g} / \mathrm{cm}^{3}$
\end{tabular}




\begin{tabular}{|c|c|c|}
\hline$\mu$ & $13.129 \mathrm{~mm}^{-1}$ & \\
\hline Data collection range & $2.875^{\circ}<\theta<27.585^{\circ}$ & $3.0260^{\circ}<\theta<28.1130^{\circ}$ \\
\hline Reflections collected & 15655 & 109759 \\
\hline Independent reflections & 2739 & 5687 \\
\hline Cell determining reflections & 1705 & 39104 \\
\hline Parameters refined & 114 & 164 \\
\hline $\mathrm{R}_{1}, \mathrm{wR}_{2}$ & $0.0230^{\mathrm{a}}, 0.0596^{\mathrm{b}}$ & $0.0657^{\mathrm{a}}, 0.1701^{\mathrm{b}}$ \\
\hline Goodness-of-fit on $\mathrm{F}^{2}$ & 1.0487 & 1.0847 \\
\hline
\end{tabular}

Table S2. Atomic positions of (bmpy) $6\left[\mathrm{~Pb}_{3} \mathrm{Br}_{12}\right]$. All non-hydrogens were refined with anisotropic displacement parameters, while the hydrogens were refined with isotropic displacement parameters.

\begin{tabular}{cccccccc}
\hline Atom & Site & $\begin{array}{c}\text { Wyckoff } \\
\text { Position }\end{array}$ & $\boldsymbol{x}$ & $\boldsymbol{y}$ & $\boldsymbol{z}$ & $\begin{array}{c}\boldsymbol{U}_{\text {eq }}, \boldsymbol{U}_{\text {iso }} \\
\left(\AA^{2}\right)\end{array}$ & SOF \\
\hline $\mathrm{Pb}$ & $\mathrm{Pb} 1$ & $3 \mathrm{a}$ & 0.6667 & 0.3333 & 0.3333 & 0.0098 & 1 \\
$\mathrm{~Pb}$ & $\mathrm{~Pb} 2$ & $6 \mathrm{c}$ & 0.6667 & 0.3333 & $0.516623(14)$ & 0.0106 & 1 \\
$\mathrm{Br}$ & $\mathrm{Br} 3$ & $18 \mathrm{f}$ & $0.66999(3)$ & $0.48190(3)$ & $0.58262(2)$ & 0.0158 & 1 \\
$\mathrm{Br}$ & $\mathrm{Br} 4$ & $18 \mathrm{f}$ & $0.78140(3)$ & $0.48829(3)$ & $0.41681(2)$ & 0.0126 & 1 \\
$\mathrm{~N}$ & $\mathrm{~N} 111$ & $18 \mathrm{f}$ & $0.3988(3)$ & $0.3935(3)$ & $0.60244(19)$ & 0.0122 & 1 \\
$\mathrm{C}$ & $\mathrm{C} 117$ & $18 \mathrm{f}$ & $0.4488(3)$ & $0.3963(3)$ & $0.6604(2)$ & 0.0129 & 1 \\
$\mathrm{C}$ & $\mathrm{C} 118$ & $18 \mathrm{f}$ & $0.4290(3)$ & $0.3082(3)$ & $0.6879(2)$ & 0.0138 & 1 \\
$\mathrm{C}$ & $\mathrm{C} 116$ & $18 \mathrm{f}$ & $0.4135(4)$ & $0.3460(3)$ & $0.5496(2)$ & 0.0144 & 1 \\
$\mathrm{C}$ & $\mathrm{C} 115$ & $18 \mathrm{f}$ & $0.3382(4)$ & $0.3251(4)$ & $0.5050(2)$ & 0.0176 & 1 \\
$\mathrm{C}$ & $\mathrm{C} 114$ & $18 \mathrm{f}$ & $0.2631(4)$ & $0.3244(4)$ & $0.5443(2)$ & 0.0177 & 1 \\
$\mathrm{C}$ & $\mathrm{C} 113$ & $18 \mathrm{f}$ & $0.2993(3)$ & $0.3434(3)$ & $0.6099(2)$ & 0.0147 & 1 \\
$\mathrm{C}$ & $\mathrm{C} 112$ & $18 \mathrm{f}$ & $0.4287(4)$ & $0.4880(3)$ & $0.5846(2)$ & 0.017 & 1 \\
$\mathrm{C}$ & $\mathrm{C} 120$ & $18 \mathrm{f}$ & $0.4892(4)$ & $0.2377(4)$ & $0.7610(2)$ & 0.0221 & 1 \\
$\mathrm{C}$ & $\mathrm{C} 119$ & $18 \mathrm{f}$ & $0.5041(4)$ & $0.3241(4)$ & $0.7315(2)$ & 0.0187 & 1 \\
$\mathrm{H}$ & $\mathrm{H} 61$ & $18 \mathrm{f}$ & 0.4357 & 0.4274 & 0.6909 & 0.0159 & 1 \\
$\mathrm{H}$ & $\mathrm{H} 62$ & $18 \mathrm{f}$ & 0.5107 & 0.4286 & 0.6511 & 0.0159 & 1
\end{tabular}




\begin{tabular}{cccccccc}
$\mathrm{H}$ & $\mathrm{H} 71$ & 18f & 0.3742 & 0.2825 & 0.7098 & 0.0174 & 1 \\
$\mathrm{H}$ & $\mathrm{H} 72$ & 18f & 0.425 & 0.2689 & 0.6559 & 0.0174 & 1 \\
$\mathrm{H}$ & $\mathrm{H} 81$ & 18f & 0.4108 & 0.2926 & 0.5634 & 0.0175 & 1 \\
$\mathrm{H}$ & $\mathrm{H} 82$ & $18 \mathrm{f}$ & 0.4698 & 0.3833 & 0.5308 & 0.0175 & 1 \\
$\mathrm{H}$ & $\mathrm{H} 91$ & $18 \mathrm{f}$ & 0.3575 & 0.3697 & 0.474 & 0.0217 & 1 \\
$\mathrm{H}$ & $\mathrm{H} 92$ & $18 \mathrm{f}$ & 0.3177 & 0.2685 & 0.4863 & 0.0217 & 1 \\
$\mathrm{H}$ & $\mathrm{H} 101$ & $18 \mathrm{f}$ & 0.2511 & 0.3694 & 0.5306 & 0.0225 & 1 \\
$\mathrm{H}$ & $\mathrm{H} 102$ & $18 \mathrm{f}$ & 0.2102 & 0.2679 & 0.5419 & 0.0225 & 1 \\
$\mathrm{H}$ & $\mathrm{H} 111$ & $18 \mathrm{f}$ & 0.2802 & 0.3788 & 0.6312 & 0.0184 & 1 \\
$\mathrm{H}$ & $\mathrm{H} 112$ & $18 \mathrm{f}$ & 0.2805 & 0.2894 & 0.6318 & 0.0184 & 1 \\
$\mathrm{H}$ & $\mathrm{H} 121$ & $18 \mathrm{f}$ & 0.3984 & 0.4886 & 0.5482 & 0.0213 & 1 \\
$\mathrm{H}$ & $\mathrm{H} 122$ & $18 \mathrm{f}$ & 0.4154 & 0.5162 & 0.6171 & 0.0213 & 1 \\
$\mathrm{H}$ & $\mathrm{H} 123$ & $18 \mathrm{f}$ & 0.4912 & 0.5191 & 0.5773 & 0.0213 & 1 \\
$\mathrm{H}$ & $\mathrm{H} 131$ & $18 \mathrm{f}$ & 0.5371 & 0.2497 & 0.788 & 0.0279 & 1 \\
$\mathrm{H}$ & $\mathrm{H} 132$ & $18 \mathrm{f}$ & 0.4349 & 0.2112 & 0.7835 & 0.0279 & 1 \\
$\mathrm{H}$ & $\mathrm{H} 133$ & $18 \mathrm{f}$ & 0.4858 & 0.198 & 0.7295 & 0.0279 & 1 \\
$\mathrm{H}$ & $\mathrm{H} 11$ & $18 \mathrm{f}$ & 0.5076 & 0.3636 & 0.7632 & 0.0232 & 1 \\
$\mathrm{H}$ & $\mathrm{H} 12$ & $18 \mathrm{f}$ & 0.5586 & 0.3504 & 0.7092 & 0.0232 & 1 \\
\hline
\end{tabular}

Table S3. Atomic positions of (bmpy) ${ }_{9}\left[\mathrm{ZnBr}_{4}\right]_{2}\left[\mathrm{~Pb}_{3} \mathrm{Br}_{11}\right]$.

\begin{tabular}{cccccc}
\hline Atom & Label & $\mathbf{x}$ & $\mathbf{y}$ & $\mathbf{z}$ & $\mathbf{U}_{\text {iso }}\left(\AA^{\mathbf{2}}\right)$ \\
\hline $\mathrm{Pb}$ & $\mathrm{Pb} 1$ & 1.4819 & 1.241 & 0.2462 & 0.0294 \\
$\mathrm{Br}$ & $\mathrm{Br} 2$ & 1.3309 & 1.1675 & 0.3151 & 0.0744 \\
$\mathrm{Zn}$ & $\mathrm{Zn} 3$ & 1.3333 & 1.6667 & $0.0416(3)$ & 0.0673 \\
$\mathrm{Br}$ & $\mathrm{Br} 4$ & 1.2452 & 1.4901 & 0.0119 & 0.0717 \\
$\mathrm{Br}$ & $\mathrm{Br} 5$ & 1.3333 & 1.6667 & $0.1193(3)$ & 0.2369 \\
$\mathrm{Br}$ & $\mathrm{Br} 6$ & 1.6667 & 1.3333 & $0.1812(2)$ & 0.0272 \\
$\mathrm{Br}$ & $\mathrm{Br} 7$ & 1.532 & 1.468 & 0.2461 & 0.0463 \\
$\mathrm{Br}$ & $\mathrm{Br} 8$ & 1.3329 & 1.1647 & 0.1772 & 0.0612 \\
$\mathrm{Br}$ & $\mathrm{Br} 9$ & 1.6667 & 1.3333 & $0.3125(2)$ & 0.0363 \\
$\mathrm{Zn}$ & $\mathrm{Zn} 1$ & 1.3333 & 1.6667 & $0.4512(3)$ & 0.0721
\end{tabular}




\begin{tabular}{|c|c|c|c|c|c|}
\hline $\mathrm{Br}$ & Br10 & 1.3333 & 1.6667 & $0.3754(4)$ & 0.122 \\
\hline $\mathrm{Br}$ & Br11 & 1.5041 & 1.7524 & 0.4723 & 0.1778 \\
\hline $\mathrm{N}$ & N100 & 1.5042 & 1.4767 & 0.0959 & 0.2 \\
\hline $\mathrm{C}$ & C101 & 1.5605 & 1.4215 & 0.109 & 0.2 \\
\hline $\mathrm{C}$ & C102 & 1.5349 & 1.5691 & 0.1248 & 0.2 \\
\hline $\mathrm{C}$ & C103 & 1.6333 & 1.6576 & 0.1053 & 0.2 \\
\hline $\mathrm{C}$ & C104 & 1.6424 & 1.622 & 0.059 & 0.2 \\
\hline $\mathrm{C}$ & C105 & 1.5404 & 1.5231 & 0.0514 & 0.2 \\
\hline $\mathrm{C}$ & C106 & 1.3901 & 1.3987 & 0.0975 & 0.2 \\
\hline $\mathrm{C}$ & C107 & 1.3457 & 1.3406 & 0.0556 & 0.2 \\
\hline $\mathrm{C}$ & C108 & 1.3398 & 1.2363 & 0.058 & 0.2 \\
\hline $\mathrm{C}$ & C109 & 1.2373 & 1.153 & 0.0459 & 0.2 \\
\hline $\mathrm{N}$ & N200 & 1.5234 & 1.5044 & 0.3985 & 0.2 \\
\hline $\mathrm{C}$ & C201 & 1.574 & 1.4439 & 0.3846 & 0.2 \\
\hline $\mathrm{C}$ & C202 & 1.4315 & 1.4772 & 0.3699 & 0.2 \\
\hline $\mathrm{C}$ & C203 & 1.3438 & 1.3761 & 0.388 & 0.2 \\
\hline $\mathrm{C}$ & C204 & 1.3825 & 1.3615 & 0.433 & 0.2 \\
\hline $\mathrm{C}$ & C205 & 1.4751 & 1.467 & 0.4425 & 0.2 \\
\hline $\mathrm{C}$ & C206 & 1.6058 & 1.6179 & 0.3973 & 0.2 \\
\hline $\mathrm{C}$ & C207 & 1.7044 & 1.6471 & 0.4219 & 0.2 \\
\hline $\mathrm{C}$ & C208 & 1.7627 & 1.7629 & 0.4304 & 0.2 \\
\hline $\mathrm{C}$ & C209 & 1.8633 & 1.7984 & 0.4503 & 0.2 \\
\hline $\mathrm{N}$ & N300 & 1.1598 & 1.3247 & 0.2498 & 0.2 \\
\hline $\mathrm{C}$ & C301 & 1.103 & 1.3727 & 0.2289 & 0.2 \\
\hline $\mathrm{C}$ & C302 & 1.2589 & 1.3602 & 0.2252 & 0.2 \\
\hline $\mathrm{C}$ & C303 & 1.2196 & 1.3108 & 0.1808 & 0.2 \\
\hline $\mathrm{C}$ & C304 & 1.1135 & 1.2121 & 0.1902 & 0.2 \\
\hline $\mathrm{C}$ & C305 & 1.1031 & 1.2112 & 0.2394 & 0.2 \\
\hline $\mathrm{C}$ & C306 & 1.1741 & 1.3528 & 0.2977 & 0.2 \\
\hline $\mathrm{C}$ & C307 & 1.1264 & 1.2667 & 0.331 & 0.2 \\
\hline $\mathrm{C}$ & C308 & 1.1768 & 1.3078 & 0.3752 & 0.2 \\
\hline $\mathrm{C}$ & C309 & 1.1867 & 1.2313 & 0.4011 & 0.2 \\
\hline $\mathrm{H}$ & H1011 & 1.5392 & 1.3618 & 0.0897 & 0.24 \\
\hline $\mathrm{H}$ & H1012 & 1.6316 & 1.465 & 0.1061 & 0.24 \\
\hline $\mathrm{H}$ & H1013 & 1.5421 & 1.3959 & 0.1376 & 0.24 \\
\hline $\mathrm{H}$ & H1021 & 1.5495 & 1.5532 & 0.1532 & 0.24 \\
\hline $\mathrm{H}$ & H1022 & 1.479 & 1.582 & 0.1267 & 0.24 \\
\hline $\mathrm{H}$ & H1031 & 1.6885 & 1.6756 & 0.1218 & 0.24 \\
\hline $\mathrm{H}$ & H1032 & 1.6197 & 1.716 & 0.1021 & 0.24 \\
\hline $\mathrm{H}$ & H1041 & 1.6521 & 1.6713 & 0.0374 & 0.24 \\
\hline $\mathrm{H}$ & H1042 & 1.6973 & 1.6079 & 0.0586 & 0.24 \\
\hline $\mathrm{H}$ & H1051 & 1.4905 & 1.5367 & 0.038 & 0.24 \\
\hline $\mathrm{H}$ & H1052 & 1.5495 & 1.4763 & 0.0329 & 0.24 \\
\hline $\mathrm{H}$ & H1061 & 1.3534 & 1.4322 & 0.1057 & 0.24 \\
\hline $\mathrm{H}$ & H1062 & 1.3782 & 1.3462 & 0.1181 & 0.24 \\
\hline
\end{tabular}




\begin{tabular}{|c|c|c|c|c|c|}
\hline $\mathrm{H}$ & H1071 & 1.3898 & 1.3784 & 0.0315 & 0.24 \\
\hline $\mathrm{H}$ & H1072 & 1.2796 & 1.3417 & 0.0494 & 0.24 \\
\hline $\mathrm{H}$ & H1081 & 1.3692 & 1.226 & 0.0378 & 0.24 \\
\hline $\mathrm{H}$ & H1082 & 1.3343 & 1.2186 & 0.0859 & 0.24 \\
\hline $\mathrm{H}$ & H1091 & 1.2071 & 1.0901 & 0.0462 & 0.24 \\
\hline $\mathrm{H}$ & H1092 & 1.205 & 1.1713 & 0.0154 & 0.24 \\
\hline $\mathrm{H}$ & H1093 & 1.1702 & 1.164 & 0.0635 & 0.24 \\
\hline $\mathrm{H}$ & H2011 & 1.6306 & 1.4592 & 0.4002 & 0.24 \\
\hline $\mathrm{H}$ & H2012 & 1.5931 & 1.4604 & 0.3531 & 0.24 \\
\hline $\mathrm{H}$ & H2013 & 1.5236 & 1.371 & 0.3847 & 0.24 \\
\hline $\mathrm{H}$ & H2021 & 1.4126 & 1.5311 & 0.3724 & 0.24 \\
\hline $\mathrm{H}$ & H2022 & 1.4426 & 1.4693 & 0.3408 & 0.24 \\
\hline $\mathrm{H}$ & H2031 & 1.2816 & 1.3817 & 0.3928 & 0.24 \\
\hline $\mathrm{H}$ & H2032 & 1.3269 & 1.3211 & 0.3693 & 0.24 \\
\hline $\mathrm{H}$ & H2041 & 1.3311 & 1.3418 & 0.455 & 0.24 \\
\hline $\mathrm{H}$ & H2042 & 1.4004 & 1.308 & 0.4306 & 0.24 \\
\hline $\mathrm{H}$ & H2051 & 1.5235 & 1.4575 & 0.4608 & 0.24 \\
\hline $\mathrm{H}$ & H2052 & 1.4569 & 1.51 & 0.4556 & 0.24 \\
\hline $\mathrm{H}$ & H2061 & 1.5758 & 1.6555 & 0.4085 & 0.24 \\
\hline $\mathrm{H}$ & H2062 & 1.6249 & 1.6356 & 0.3677 & 0.24 \\
\hline $\mathrm{H}$ & H2071 & 1.6842 & 1.6105 & 0.4501 & 0.24 \\
\hline $\mathrm{H}$ & H2072 & 1.7447 & 1.6264 & 0.4071 & 0.24 \\
\hline $\mathrm{H}$ & H2081 & 1.7219 & 1.779 & 0.4498 & 0.24 \\
\hline $\mathrm{H}$ & H2082 & 1.7707 & 1.7961 & 0.4037 & 0.24 \\
\hline $\mathrm{H}$ & H2091 & 1.896 & 1.8699 & 0.4546 & 0.24 \\
\hline $\mathrm{H}$ & H2092 & 1.854 & 1.7638 & 0.4776 & 0.24 \\
\hline $\mathrm{H}$ & H2093 & 1.9028 & 1.7809 & 0.4316 & 0.24 \\
\hline $\mathrm{H}$ & H3011 & 1.0392 & 1.3511 & 0.2452 & 0.24 \\
\hline $\mathrm{H}$ & H3012 & 1.1427 & 1.4451 & 0.23 & 0.24 \\
\hline $\mathrm{H}$ & H3013 & 1.0852 & 1.3487 & 0.2002 & 0.24 \\
\hline $\mathrm{H}$ & H3021 & 1.2952 & 1.4323 & 0.2214 & 0.24 \\
\hline $\mathrm{H}$ & H3022 & 1.2999 & 1.3366 & 0.238 & 0.24 \\
\hline $\mathrm{H}$ & H3031 & 1.2053 & 1.3493 & 0.1617 & 0.24 \\
\hline $\mathrm{H}$ & H3032 & 1.2623 & 1.2873 & 0.1682 & 0.24 \\
\hline $\mathrm{H}$ & H3041 & 1.0551 & 1.2091 & 0.1782 & 0.24 \\
\hline $\mathrm{H}$ & H3042 & 1.1117 & 1.1464 & 0.1835 & 0.24 \\
\hline $\mathrm{H}$ & H3051 & 1.1342 & 1.1775 & 0.2555 & 0.24 \\
\hline $\mathrm{H}$ & H3052 & 1.0321 & 1.18 & 0.2503 & 0.24 \\
\hline $\mathrm{H}$ & H3061 & 1.2499 & 1.3926 & 0.3025 & 0.24 \\
\hline $\mathrm{H}$ & H3062 & 1.1514 & 1.4028 & 0.3023 & 0.24 \\
\hline $\mathrm{H}$ & H3071 & 1.0577 & 1.2521 & 0.335 & 0.24 \\
\hline $\mathrm{H}$ & H3072 & 1.1361 & 1.2159 & 0.3238 & 0.24 \\
\hline $\mathrm{H}$ & H3081 & 1.2523 & 1.37 & 0.3698 & 0.24 \\
\hline $\mathrm{H}$ & H3082 & 1.1475 & 1.3431 & 0.3911 & 0.24 \\
\hline $\mathrm{H}$ & H3091 & 1.2232 & 1.2686 & 0.4289 & 0.24 \\
\hline
\end{tabular}




\begin{tabular}{llllll}
$\mathrm{H}$ & $\mathrm{H} 3092$ & 1.2251 & 1.2107 & 0.3873 & 0.24 \\
$\mathrm{H}$ & $\mathrm{H} 3093$ & 1.1203 & 1.1838 & 0.4086 & 0.24 \\
\hline
\end{tabular}

Table S4. Elemental Analysis of (bmpy) $\left.6 \mathrm{~Pb}_{3} \mathrm{Br}_{12}\right]$ and (bmpy) ${ }_{9}\left[\mathrm{ZnBr}_{4}\right]_{2}\left[\mathrm{~Pb}_{3} \mathrm{Br}_{11}\right]$.

\begin{tabular}{lllll}
\hline \multicolumn{3}{c}{$\left(\mathbf{b m p y}_{\mathbf{6}}\left[\mathrm{Pb}_{\mathbf{3}} \mathbf{B r}_{12}\right]\right.$} & \multicolumn{2}{c}{$(\mathbf{b m p y})_{\mathbf{9}}\left[\mathbf{Z n B r}_{4}\right]_{2}\left[\mathbf{P b}_{3} \mathbf{B r}_{11}\right]$} \\
\hline & Theoretical & Experimental & Theoretical & Experimental \\
$\mathrm{C}$ & 26.65 & 26.89 & 27.40 & 27.66 \\
$\mathrm{H}$ & 4.97 & 4.85 & 5.11 & 5.08 \\
$\mathrm{~N}$ & 3.45 & 3.35 & 3.55 & 3.41 \\
$\mathrm{Br}$ & 39.39 & 39.64 & 42.75 & 43.04 \\
\hline
\end{tabular}

\section{References}

1. Cortecchia, D.; Neutzner, S.; Srimath Kandada, A. R.; Mosconi, E.; Meggiolaro, D.; De Angelis, F.; Soci, C.; Petrozza, A., Broadband Emission in Two-Dimensional Hybrid Perovskites: The Role of Structural Deformation. J Am Chem Soc 2017, 139 (1), 39-42.

2. Zhou, C.; Tian, Y.; Yuan, Z.; Han, M.; Wang, J.; Zhu, L.; Tameh, M. S.; Huang, C.; Ma, B., Precise Design of Phosphorescent Molecular Butterflies with Tunable Photoinduced Structural Change and Dual Emission. Angewandte Chemie International Edition 2015, 54 (33), 9591-9595. 